\title{
Singular charge fluctuations at a magnetic quantum critical point
}

\author{
L. Prochaska ${ }^{1, *}$, X. Li $i^{2, *}$, D. C. MacFarland ${ }^{1,3, *}$, A. M. Andrews ${ }^{3}$,

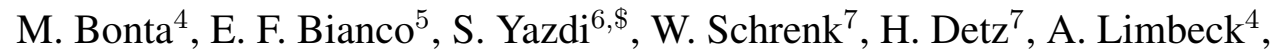

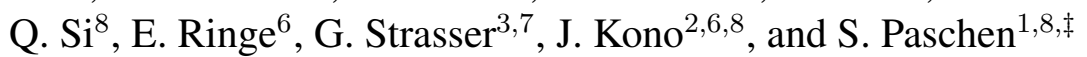 \\ ${ }^{1}$ Institute of Solid State Physics, TU Wien, Wiedner Hauptstr. 8-10, 1040 Vienna, Austria \\ ${ }^{2}$ Department of Electrical and Computer Engineering, 6100 Main Street, Rice University, Houston, \\ Texas 77005, USA \\ ${ }^{3}$ Institute of Solid State Electronics, TU Wien, Nanocenter Campus Gußhaus, Gußhausstr. 25-25a, \\ Gebäude CH, 1040 Vienna, Austria \\ ${ }^{4}$ Institute of Chemical Technologies and Analytics, TU Wien, Getreidemarkt 9, 1060 Vienna, Austria \\ ${ }^{5}$ Department of Chemistry, 6100 Main Street, Rice University, Houston, Texas 77005, USA \\ ${ }^{6}$ Department of Materials Science and Nanoengineering, 6100 Main Street, Rice University, Houston, \\ Texas 77005, USA \\ ${ }^{7}$ Center for Micro- and Nanostructures, Nanocenter Campus Gußhaus, Gußhausstr. 25-25a, Gebäude \\ CH, 1040 Vienna, Austria \\ ${ }^{8}$ Department of Physics and Astronomy, Center for Quantum Materials, 6100 Main Street, Rice \\ University, Houston, Texas 77005, USA
}

August 8, 2018

Strange metal behavior is ubiquitous in correlated materials ranging from cuprate superconductors to bilayer graphene. There is increasing recognition that it arises from physics beyond the quantum fluctuations of a Landau order parameter which, in quantum critical heavy fermion antiferromagnets, may be realized as critical Kondo entanglement of spin and charge. The dynamics of the associated electronic delocalization transition could be ideally probed by optical conductivity, but experiments in the corresponding frequency and temperature ranges have remained elusive. We present terahertz time-domain transmission spectroscopy on molecular beam epitaxy-grown thin films of $\mathbf{Y b R h}_{2} \mathrm{Si}_{2}$, a model strange metal compound. We observe frequency over temperature scaling of the optical conductivity as a hallmark of beyond-Landau quantum criticality. Our discovery implicates critical charge fluctuations as playing a central role in the strange metal behavior, thereby elucidating one of the longstanding mysteries of correlated quantum matter. 
*These authors contributed equally to this work.

\$Present address: Renewable and Sustainable Energy Institute, University of Colorado Boulder, Boulder, Colorado 80309, USA

‡To whom correspondence should be addressed; E-mail: paschen@ifp.tuwien.ac.at. 
Quantum critical behavior as prescribed by the Landau framework of order parameter fluctuations $(1,2)$ has been clearly identified in insulating quantum magnets such as $\mathrm{LiHoF}_{4}(3)$ and $\mathrm{TlCuCl}_{3}$ (4). In strongly correlated metals, however, this framework often fails. This hints at the charge carriers as giving rise to new forms of quantum criticality. In the strange metal (5) regime of various correlated systems (6), electronic localization-delocalization transitions have been evidenced (7-14) and it is an outstanding question whether they are a key ingredient of beyond-Landau quantum criticality. To make progress, it is essential to study the dynamics of charge carriers in a suitable setting.

We chose the heavy fermion metal $\mathrm{YbRh}_{2} \mathrm{Si}_{2}$ (15) for our investigation because it has a well-defined quantum critical point $(15,16)$ and shows evidence for an electron delocalization transition $(7,8)$ in its strange metal regime. An ideal tool for such studies are optical conductivity measurements in the relevant frequency window, which is typically the terahertz $(\mathrm{THz})$ range and below for heavy fermion systems. However, such measurements are challenging on bulk samples because the Kramers-Kronig transformation to extract the real and imaginary parts of the optical conductivity from reflectivity measurements introduces substantial uncertainty at low frequencies (17). Thus, we resorted to an entirely different and previously unattempted approach: We performed $\mathrm{THz}$ time-domain transmission spectroscopy experiments on thin films of $\mathrm{YbRh}_{2} \mathrm{Si}_{2}$ grown by molecular beam epitaxy (MBE). Our measurements reveal $\omega / T$ scaling of the optical conductivity, thereby elucidating the mechanism for strange metal phenomena.

To grow epitaxial thin films of $\mathrm{YbRh}_{2} \mathrm{Si}_{2}$ on (THz transparent) Ge substrates (Fig. 11A), we used a specially equipped MBE system (18). The epitaxial growth of phase-pure $\mathrm{YbRh}_{2} \mathrm{Si}_{2}$ is confirmed by X-ray diffraction (Fig.11B) (18), and the high quality of the film and the filmsubstrate interface are revealed by high-resolution transmission electron microscopy (Fig.11C, D) (18). The temperature dependence of the (quasi) (18) dc electrical resistivity $\rho(T)$ of these films (18) is similar to that of bulk single crystals $(15,19)$ (Fig.22). Most notably, it displays 
strange metal behavior, $\rho=\rho_{0}+A^{\prime} T^{\alpha}$ (Fig. 2B), with an exponent $\alpha$ that strongly deviates from the Fermi liquid value $\alpha=2$, and tends to $\alpha=1$ in the low-temperature limit (Fig. $2 \mathrm{C}$ ).

The frequency dependence of the real part of the complex optical conductivity, $\operatorname{Re}(\sigma)$, measured at temperatures between 1.4 and $250 \mathrm{~K}$ and frequencies between 0.25 and $2.6 \mathrm{THz}$, is shown in Fig. 3A (see Fig. S1 for the imaginary part, $\operatorname{Im}(\sigma)$ ). The de electrical conductivity $\sigma=1 / \rho$ values, plotted as symbols at $\omega=0$, are compatible with the extrapolation of the finite frequency results to zero frequency. $\operatorname{Both} \operatorname{Re}(\sigma)$ and $\operatorname{Im}(\sigma)$ are flat and featureless at temperatures above about $80 \mathrm{~K}$, indicating strong incoherent scattering of charges, but develop sizeable temperature and frequency dependence at lower temperatures, with spectral weight of $\operatorname{Re}(\sigma)$ being transferred to low frequencies. The increasingly sharp and pronounced resonance of $\operatorname{Re}(\sigma)$, with non-Lorentzian shape, may, in clean samples, be associated with non-Fermi liquid behavior. In the far-infrared range, where there is overlap with data from optical reflectivity measurements on bulk single crystals (20), the agreement is good and our results confirm the deviations from a simple Drude response discussed there.

To explore dynamical scaling, we plot $\operatorname{Re}[\sigma(\omega)] \cdot T^{\alpha}$ as a function of $\hbar \omega /\left(k_{\mathrm{B}} T\right)$ for temperatures $(T \leq 15 \mathrm{~K})$ well below the material's Kondo temperature $T_{\mathrm{K}}=24 \mathrm{~K}$ (15) (Fig. $\left.3 \mathrm{~B}\right)$ and frequencies below $2 \mathrm{THz}$. Indeed, for $\alpha=0.74$, all curves collapse, demonstrating $\omega / T$ scaling of $\operatorname{Re}[\sigma(\omega)]$. This is the first time $\omega / T$ scaling of the charge carrier response is directly observed in $\mathrm{YbRh}_{2} \mathrm{Si}_{2}$ and, to the best of our knowledge, in any optical conductivity measurement on quantum critical heavy fermion compounds.

The critical exponent $\alpha$ is somewhat smaller than that of the dc electrical resistivity in the $T \rightarrow 0$ limit, which is close to 1 (Fig. $2 \mathrm{C}$ ). Note, however, that both our thin film and bulk single crystals $(15,19)$ show deviations from 1 at somewhat higher temperatures. In particular, the exponent falls below 0.9 above about $8 \mathrm{~K}$ for our thin film (Fig. 2 $\mathrm{C})$. Indeed, when limiting the scaling to temperatures below $5 \mathrm{~K}$, we obtain $\alpha=0.83$ (Fig. S2). If, in addition, we limit the 
frequency range to below $0.7 \mathrm{THz}$, which corresponds to a thermal energy of about $5 \mathrm{~K}$ (18), the exponent reaches 0.94 . Given that $1 / \rho$ is the $\omega \rightarrow 0$ limit of $\operatorname{Re}(\sigma)$, the exponent in the dynamical scaling function of $\operatorname{Re}(\sigma)$ should be compatible with that of $\rho(T)$. Within the error bars of about $10 \%$ for the experimental determination of $\alpha$, this is indeed the case.

How can the optical conductivity, which probes charge fluctuations, show $\omega / T$ scaling at an antiferromagnetic quantum critical point where, a priori, only spin fluctuations are expected - and indeed observed (21-23) - to be critical? A natural way for this to happen is to have a critical form of the Kondo entanglement between the local moments and the conduction electrons (24-26), as illustrated in Fig. 4. Across the quantum critical point, the conduction electrons go from being (asymptotically) decoupled from the local moments (bottom left box) to being entangled with them (bottom right box). Correspondingly, the elementary excitations change from single conduction electrons/holes and separated spin excitations (top left box) to the heavy quasiparticles (top right box), hybrids of the slow composite fermions (large tadpole) and the bare conduction electrons (small tadpole). In other words, the single electron excitations capture the continuous onset of the Kondo entanglement at the quantum critical point and are part of the critical degrees of freedom. Thus, optical conductivity, which probes the charge current of the elementary excitations, manifests the singular fluctuations of the QCP. In fact, within the Landau description of a metallic antiferromagnetic quantum critical point $(1,2)$, the slow long-wavelength fluctuations of the order parameter alone describe a Gaussian fixed point, where $\omega / T$ scaling is violated. The incorporation of the single electron excitations in the quantum critical spectrum not only makes charge fluctuations part of the quantum criticality, but also turns the fixed point into an interacting one (24), thereby leading to $\omega / T$ scaling.

Dynamical scaling of the optical conductivity in the region of $T$-linear resistivity has also been analysed in an optimally doped Bi-2212 cuprate (27). There, different scaling functions are needed in different $\omega / T$ ranges, leaving it open how the fluctuations of the charge carri- 
ers connect with the robust linear-in-temperature resistivity of the cuprate superconductors. By contrast, in the present study of $\mathrm{YbRh}_{2} \mathrm{Si}_{2}$, a single $\omega / T$ scaling form is uncovered in its strange metal regime. It is important to explore the dynamical scaling of the optical conductivity in other materials classes with strange metal behavior; one can then assess whether the charge carrier dynamics emerging from a localization-delocalization quantum critical point, as proposed here, is a universal mechanism of strange metal behavior. Our scaling results also provide an intriguing link to the quantum scaling of metal-insulator transitions, both in Mott-Hubbard (28-30) and in disordered systems (31).

To summarize, we have discovered the dynamical scaling of charge fluctuations at an antiferromagnetic quantum critical point. This long-sought-after effect was made possible by mastering the challenging MBE thin-film growth of a strange metal, which allowed for the application of $\mathrm{THz}$ time-domain transmission spectroscopy into the quantum critical frequency range. Our results demonstrate the charge carriers to be a central ingredient of the singular physics at the border of antiferromagnetic order, thereby providing direct evidence for the beyond-Landau nature of metallic quantum criticality. Our findings also delineate the role of electronic localization transitions in strange metal phenomena, which appear to be central to the cuprate superconductors, bilayer graphene, and a variety of other strongly correlated materials (32) and beyond (33).

\section{Acknowledgments}

We thank P. Gegenwart, Y.-B. Kim, H. von Löhneysen, S. Nakatsuji, H.-C. Nägerl, and A. Prokofiev for useful discussions. Financial support for this work was provided by the European Research Council (ERC Advanced Grant 227378), the U.S. Army Research Office (ARO W911NF-14-1-0496), and the Austrian Science Fund (FWF W1243 and P29279-N27). X.L. and J.K. acknowledge financial support from the National Science Foundation (NSF MRSEC DMR-1720595) and the ARO (W911NF-17-1-0259). Q.S. acknowledges financial support from 
the NSF (DMR-1611392), the Robert A. Welch Foundation (C-1411), and the ARO (W911NF14-1-0525), and hospitality of the University of California at Berkeley, the Aspen Center for Physics (NSF grant PHY-1607611), and the Los Alamos National Laboratory (via a Ulam Scholarship from the Center for Nonlinear Studies).

\section{References}

1. J. Hertz, Phys. Rev. B 14, 1165 (1976).

2. A. J. Millis, Phys. Rev. B 48, 7183 (1993).

3. D. Bitko, T. F. Rosenbaum, G. Aeppli, Phys. Rev. Lett. 77, 940 (1996).

4. P. Merchant, et al., Nat. Phys. 10, 373 (2014).

5. P. Giraldo-Gallo, et al., Science 361, 479 (2018).

6. J. A. N. Bruin, H. Sakai, R. S. Perry, A. P. Mackenzie, Science 339, 804 (2013).

7. S. Paschen, et al., Nature 432, 881 (2004).

8. S. Friedemann, et al., Proc. Natl. Acad. Sci. USA 107, 14547 (2010).

9. J. Custers, et al., Nat. Mater. 11, 189 (2012).

10. Y. Luo, et al., Nat. Mater. 13, 777 (2014).

11. F. F. Balakirev, et al., Nature 424, 912 (2003).

12. H. Oike, K. Miyagawa, H. Taniguchi, K. Kanoda, Phys. Rev. Lett. 114, 067002 (2015).

13. S. Badoux, et al., Nature 531, 210 (2016).

14. Y. Cao, et al., Nature 556, 43 (2018). 
15. O. Trovarelli, et al., Phys. Rev. Lett. 85, 626 (2000).

16. P. Gegenwart, et al., Phys. Rev. Lett. 89, 056402 (2002).

17. J. Orenstein, et al., Phys. Rev. B 42, 6342 (1990).

18. Materials and methods are available as supplementary materials.

19. T. Westerkamp, Quantenphasenübergänge in den Schwere-Fermionen-Systemen $\mathrm{Yb}\left(\mathrm{Rh}_{1-x} \mathrm{M}_{x}\right)_{2} \mathrm{Si}_{2}$ und $\mathrm{CePd}_{1-x} \mathrm{Rh}_{x}$, Ph.D. thesis, TU Dresden, Germany (2009). Unpublished.

20. S. Kimura, et al., Phys. Rev. B 74, 132408 (2006).

21. M. Aronson, et al., Phys. Rev. Lett. 75, 725 (1995).

22. A. Schröder, et al., Nature 407, 351 (2000).

23. C. Stock, et al., Phys. Rev. Lett. 109, 127201 (2012).

24. Q. Si, S. Rabello, K. Ingersent, J. Smith, Nature 413, 804 (2001).

25. P. Coleman, C. Pépin, Q. Si, R. Ramazashvili, J. Phys.: Condens. Matter 13, R723 (2001).

26. T. Senthil, M. Vojta, S. Sachdev, Phys. Rev. B 69, 035111 (2004).

27. D. van der Marel, et al., Nature 425, 271 (2003).

28. T. Senthil, Phys. Rev. B 78, 035103 (2008).

29. H. Terletska, J. Vučičević, D. Tanasković, V. Dobrosavljević, Phys. Rev. Lett. 107, 026401 (2011).

30. T. Furukawa, K. Miyagawa, H. Taniguchi, R. Kato, K. Kanoda, Nat. Phys. 11, 221 (2015). 
31. H.-L. Lee, J. P. Carini, D. V. Baxter, W. Henderson, G. Grüner, Science 287, 633 (2000).

32. B. Keimer, J. E. Moore, Nat. Phys. 13, 1045 (2017).

33. P. T. Brown, et al., Bad metallic transport in a cold atom Fermi-Hubbard system, arXiv:1802.09456. 
A

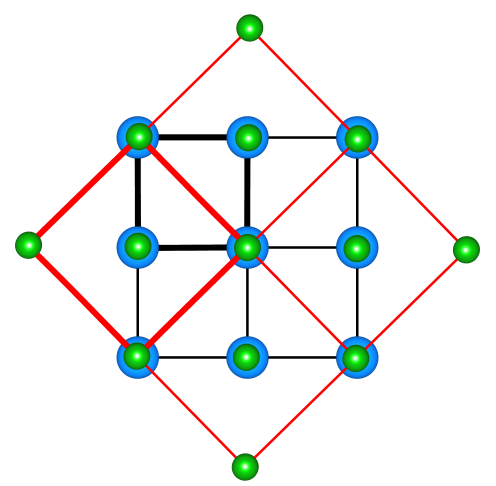

C
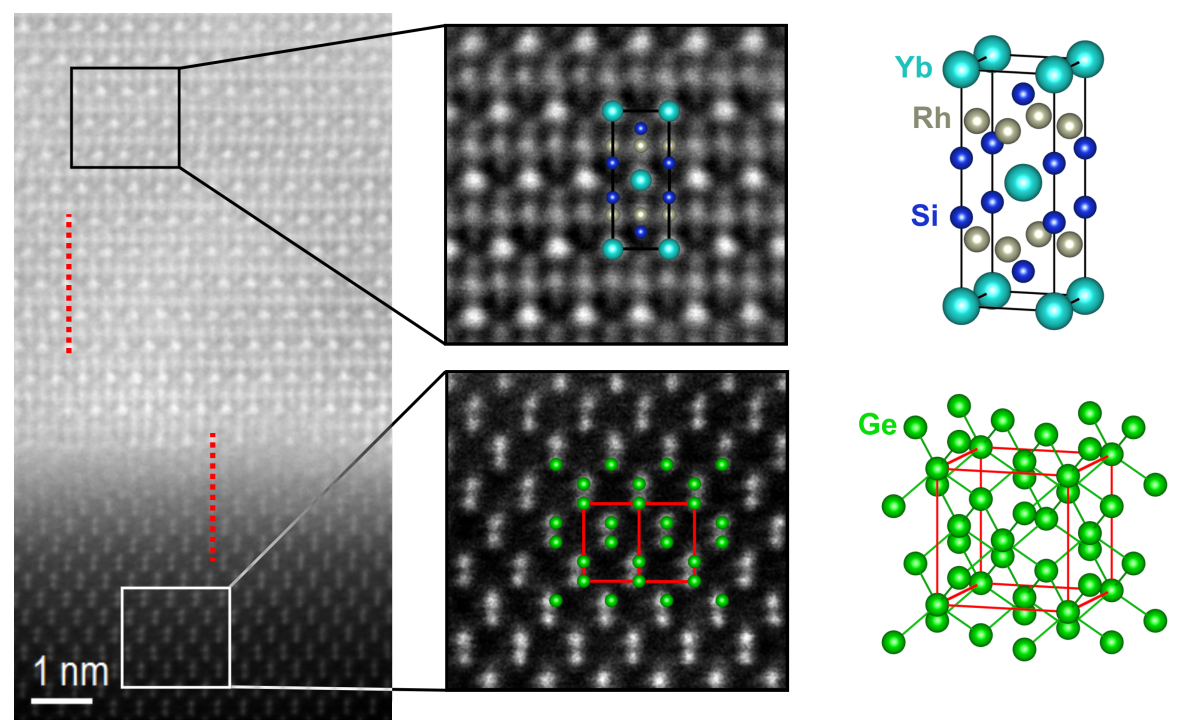

B

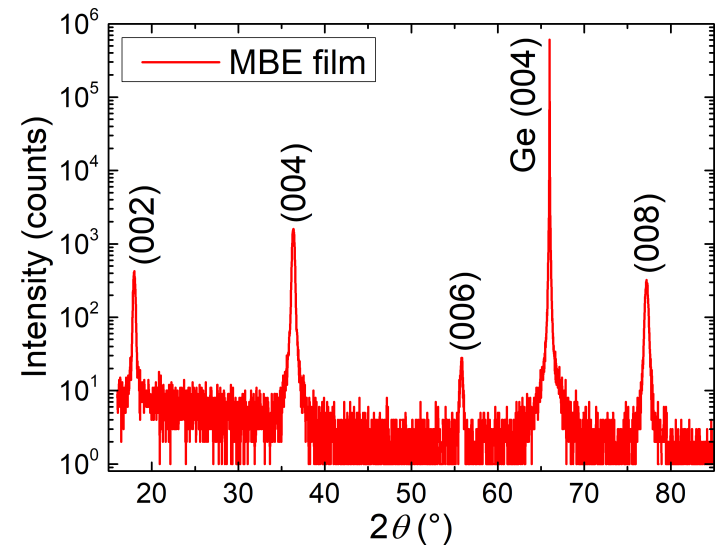

D
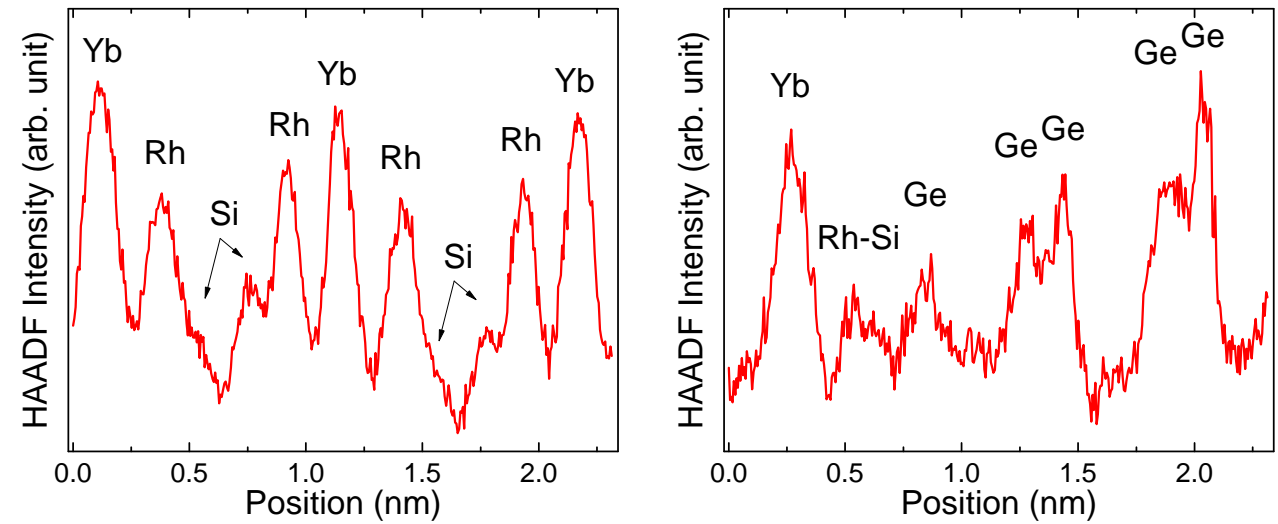
Figure 1: $\mathbf{Y b R h}{ }_{2} \mathbf{S i}_{2}$ thin films grown by molecular beam epitaxy. (A) Visualization of the lattice matching between $\mathrm{YbRh}_{2} \mathrm{Si}_{2}$ (blue circles, black lines) and $\mathrm{Ge}$ (green circles, red lines), with the crystallographic $c$ directions pointing out of the plane. For the $\mathrm{Yb}$ atoms to associate with the Ge atoms, the respective unit cells (thick lines, see panel $\mathbf{C}$ right) are rotated by $45^{\circ}$ with respect to each other around the $c$ direction. (B) High-resolution X-ray diffraction pattern, confirming that the film is phase pure $\mathrm{YbRh}_{2} \mathrm{Si}_{2}$. (C) Atomic resolution high-angle annular dark field scanning transmission electron microscopy (HAADF-STEM) image of the interface between film (top left) and substrate (bottom left), representative enlarged views with simulated overlays (center), and the corresponding unit cells in their relaxed states (right). (D) Intensity profiles along the red dashed lines in ( $\mathbf{C}$ left), within the film (upper line, left panel) and across the interface (lower line, right panel). 
A
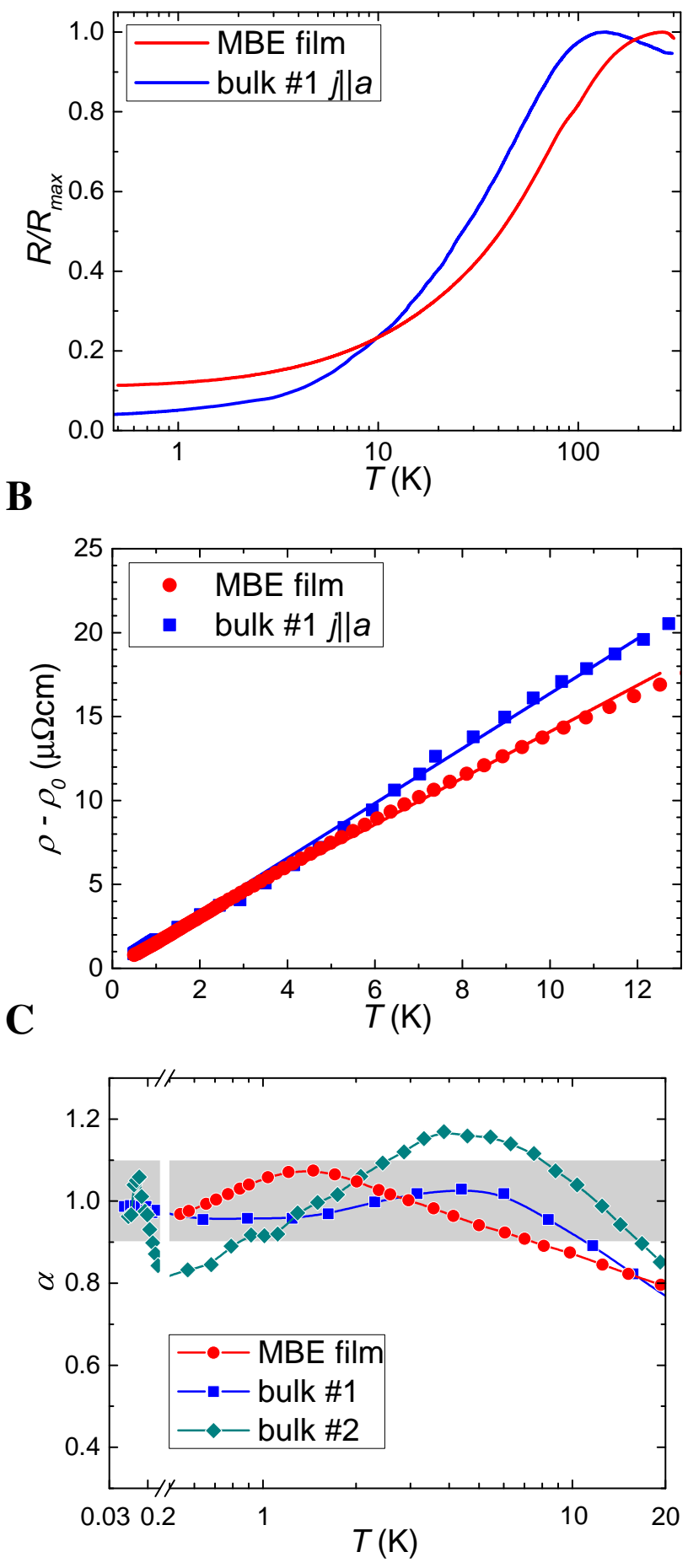
Figure 2: Electrical resistivity of MBE-grown $\mathbf{Y b R h}_{2} \mathbf{S i}_{2}$. (A) Normalized resistance of an MBE-grown $\mathrm{YbRh}_{2} \mathrm{Si}_{2}$ film, and a bulk single crystal with current $j$ within the tetragonal $a a$ plane (15) for comparison. The film was measured using the van der Pauw technique. (B) Corresponding low-temperature resistivities, with the residual resistivities $\left(\rho_{0}=11.6 \mu \Omega \mathrm{cm}\right.$ and $2.45 \mu \Omega \mathrm{cm}$ for the MBE and bulk (15) samples, respectively, determined by linear-in- $T$ fits to the data below $1 \mathrm{~K}$ ) subtracted, displaying non-Fermi liquid behavior (lines represent $\rho-\rho_{0}=$ $A^{\prime} T^{\alpha}$ fits with constant $\alpha$ to the data below $12 \mathrm{~K}$ ). (C) Temperature-dependent exponent $\alpha$, determined as $\partial \log \left(\rho-\rho_{0}\right) / \partial \log (T)$ for the two above samples, and an additional bulk single crystal (\#2, $\left.\rho_{0}=0.5 \mu \Omega \mathrm{cm}\right)$ (19) for comparison. The exponent approaches 1 in the lowtemperature limit, but falls below 0.9 above about $8 \mathrm{~K}$ for our thin film, and above 11 and $16 \mathrm{~K}$ for two different bulk single crystals $(15,19)$. Within the experimental error bar of $\Delta \alpha \approx 10 \%$, all data points below $15 \mathrm{~K}$ fall in a zone of $\alpha=1.0 \pm 0.1$ (grey shading). 
A

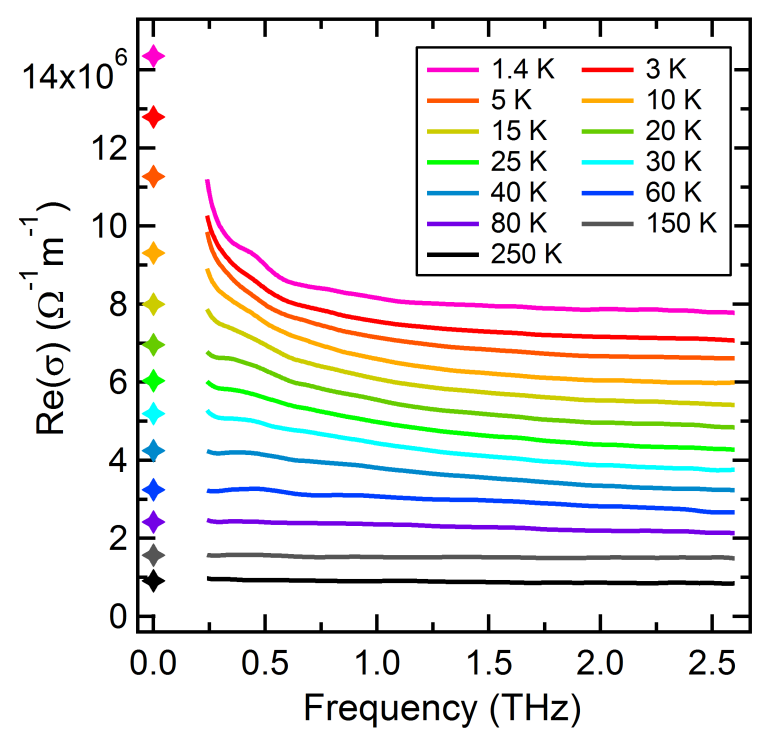

B

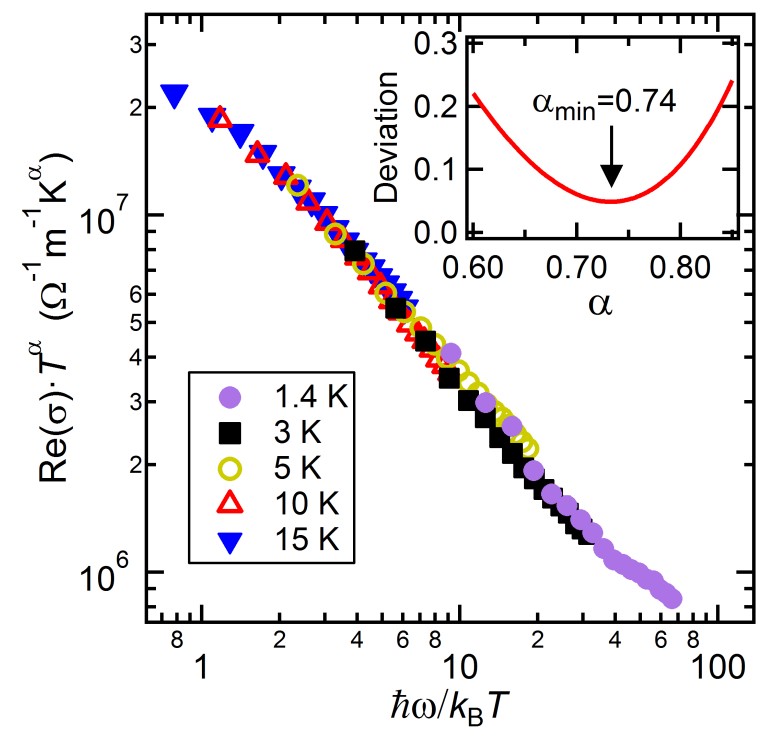

Figure 3: THz time-domain transmission spectroscopy of MBE-grown $\mathbf{Y b R h}_{2} \mathbf{S i}_{2}$. (A) Real part of optical conductivity $\operatorname{Re}(\sigma)$ vs frequency at different temperatures, with corresponding dc values marked as zero-frequency points. Curves below $250 \mathrm{~K}$ are successively offset by $6 \times 10^{5} \Omega^{-1} m^{-1}$ for clarity. (B) $\omega / T$ scaling, with a critical exponent of $\alpha=0.74$, revealed by curves of $\operatorname{Re}[\sigma(\omega)] \cdot T^{\alpha}$ as a function of $\hbar \omega /\left(k_{\mathrm{B}} T\right)$ collapsing onto a single curve for temperatures $T \leq 15 \mathrm{~K}$ and frequencies below $2 \mathrm{THz}$. (B inset) Normalized deviation between curves as a function of $\alpha$, revealing best scaling for $\alpha=0.74$. 

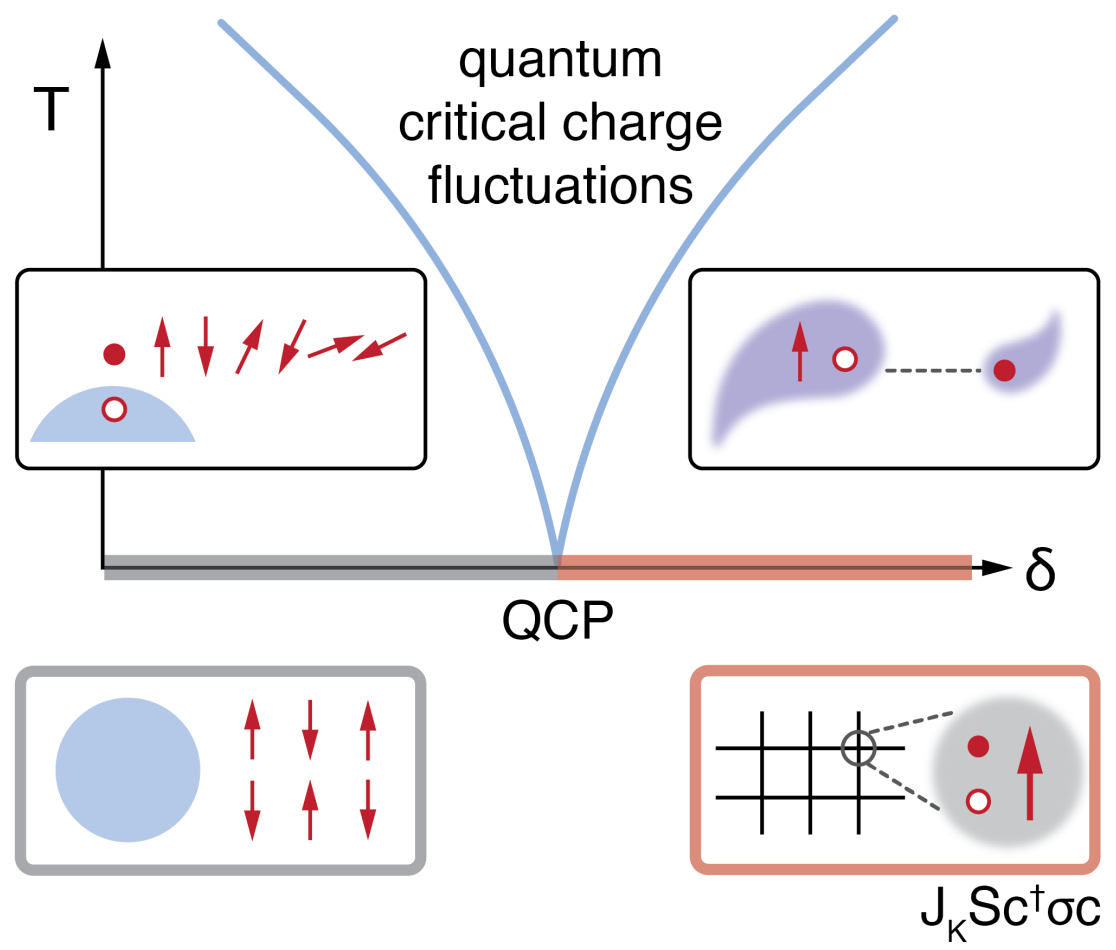

Figure 4: Sketch of quantum critical charge fluctuations emerging from Kondo disentanglement. Tuning a heavy fermion metal with a non-thermal parameter $\delta$, which microscopically corresponds to the ratio of Kondo to RKKY coupling, from an antiferromagnetic ground state with local moment order (bottom left box; circle and arrows represent Fermi sphere and local moments, respectively) to a Kondo entangled paramagnet (bottom right box; the antiferromagnetic Kondo exchange $J_{K}$ favors the formation of a Kondo singlet between the local moment $S$, represented as arrow, and the spin of the conduction electrons $c^{\dagger} \sigma c$, i.e., the particle-hole excitation of the Fermi sea in the spin-triplet channel) creates quantum critical charge fluctuations in the excitation spectrum (top boxes, see main text). 